\title{
Intraventricular atypical teratoid rhabdoid tumour in an adult: a case report and literature review
}

\author{
Vivien Chan ${ }^{1}$, Alessandro Marro ${ }^{2}$, Jonathan Chainey ${ }^{1}$, Laura M Schmitt ${ }^{1}$, Sumit Das ${ }^{1}$ \\ ${ }^{1}$ University of Alberta, Edmonton, Canada, ${ }^{2}$ University of Toronto, Toronto, Canada
}

\begin{abstract}
We report a case of atypical teratoid/rhabdoid tumour (AT/RT) in an adult patient in the deep grey matter with extension into the lateral ventricle. To our knowledge, this is the first example of AT/RT involving the lateral ventricle in an adult. The patient presented with headache and confusion, and subsequently required emergent surgery. His postoperative course was complicated by hemorrhage into the surgical site. The location and vascularity of the tumour affected the extent of resection achieved and likely contributed to postoperative complications. We discuss radiological features of AT/RT in adults and implications for investigations and management.
\end{abstract}

Key words: atypical teratoid/rhabdoid tumour, CNS tumour, intraventricular, adult, case report.

\section{Introduction}

Atypical teratoid/rhabdoid tumour (AT/RT) is a malignant neoplasm representing less than $5 \%$ of central nervous system tumours [7,11]. Although this tumour is typically seen in the pediatric population, cases of AT/RT have been reported in adults [2]. The most common locations in adults are the cerebral hemisphere and the sellar region $[2,18]$. We report a case of AT/RT in an adult patient found in the right lateral ventricle with extension into the deep grey structures. To our knowledge, this is the first case report of AT/RT in an adult in this location and involving the deep grey matter. Additionally, we discuss the radiological features of AT/RT in adults and implications for pre-operative investigations and management.

\section{Case report}

\section{Clinical presentation}

Our patient is a 27 -year-old male who presented to the hospital after a 4-week history of pulsa- tile right frontal headache with episodes of confusion. Prior to his admission, he had presented to the emergency department three times in 11 days with a primary complaint of headache.

His past medical history was significant for an episode of unprovoked generalized tonic-clonic seizure at 16 years of age. At the time of the seizure, no investigations were done and his neurological exam was documented as normal. He also had a history of sleep apnea and asthma. He had no significant family history or psychosocial history.

On examination, the patient was somnolent but easily arousable. He was confused to time and place. Due to his agitation, a comprehensive neurological exam could not be completed. Examination of his cranial nerves was unremarkable. He had a mild left pronator drift with no obvious motor weakness on examination. He denied any sensory changes. 


\section{Neuroradiology}

The computed tomography (CT) of the head revealed a heterogeneous hyperattenuated intra-axial mass within the right basal ganglia, internal capsule, and thalamus measuring $5.0 \times 4.5 \mathrm{~cm}$ with extension into the right lateral ventricle (Fig. 1).

The magnetic resonance imaging (MRI) showed a mixed cystic/necrotic mass $(3.7 \times 4.4 \times 4.1 \mathrm{~cm})$ with mixed irregular enhancement (Fig. 2). The mass centered in the region of the right thalamus and extended laterally through the corona radiata, posterior limb of the internal capsule, and right lentiform nucleus. It extended medially into the right lateral ventricle. A rim of edema surrounding the tumour was noted. The solid components of the mass demonstrated restricted diffusion. On susceptibility-weighted imaging, there were several small foci of hyperintense signal, suggestive of blood products. Given the intraventricular location, the differential diagnoses included extraventricular neurocytoma with ventricular extension, primary central neurocytoma, and ependymoma. Due to the intraparenchymal component of the tumour, along with features suggestive of a malignant neoplasm such as the heterogeneous enhancement, areas of necrosis and edema, anaplastic astrocytoma and glioblastoma multiforme were also on the differential diagnosis. Spine imaging was not obtained due to the patient's deteriorating clinical status.

\section{Surgical management}

The patient was taken to the operative room two days after his admission due to signs of raised intra- cranial pressure. A right frontal craniotomy for interhemispheric transcallosal internal decompression of an intraventricular and periventricular tumour was performed. The tumour appeared multi-coloured, multi-textured, necrotic, and extremely vascular. A partial resection was achieved due to difficulty in reaching the edge of tumour within the left lateral ventricle and the vascular nature of the tumour.

\section{Postoperative course}

An immediate postoperative CT scan showed hemorrhage at the tumour site. On postoperative day one, the patient had a sudden decrease in the level of consciousness. A repeat CT scan showed an increase in the amount of hemorrhage at the tumour site. He continued to deteriorate and by postoperative day three his examination was consistent with a diagnosis of brain death by neurological criteria. A decision was made to withdraw care.

\section{Neuropathological examination}

Intraoperative smear preparation revealed several rhabdoid cells (Fig. 3). Neuropathological examination of the permanent sections revealed a high-grade neoplasm consisting of sheets of rhabdoid tumour cells characterized by eccentrically located nuclei containing vesicular chromatin along with abundant eosinophilic cytoplasm and noticeable globular eosinophilic inclusions (Fig. 3). The tumour also showed areas of necrosis and frequent mitosis and apoptosis. Tumour cells shows scattered immuno-

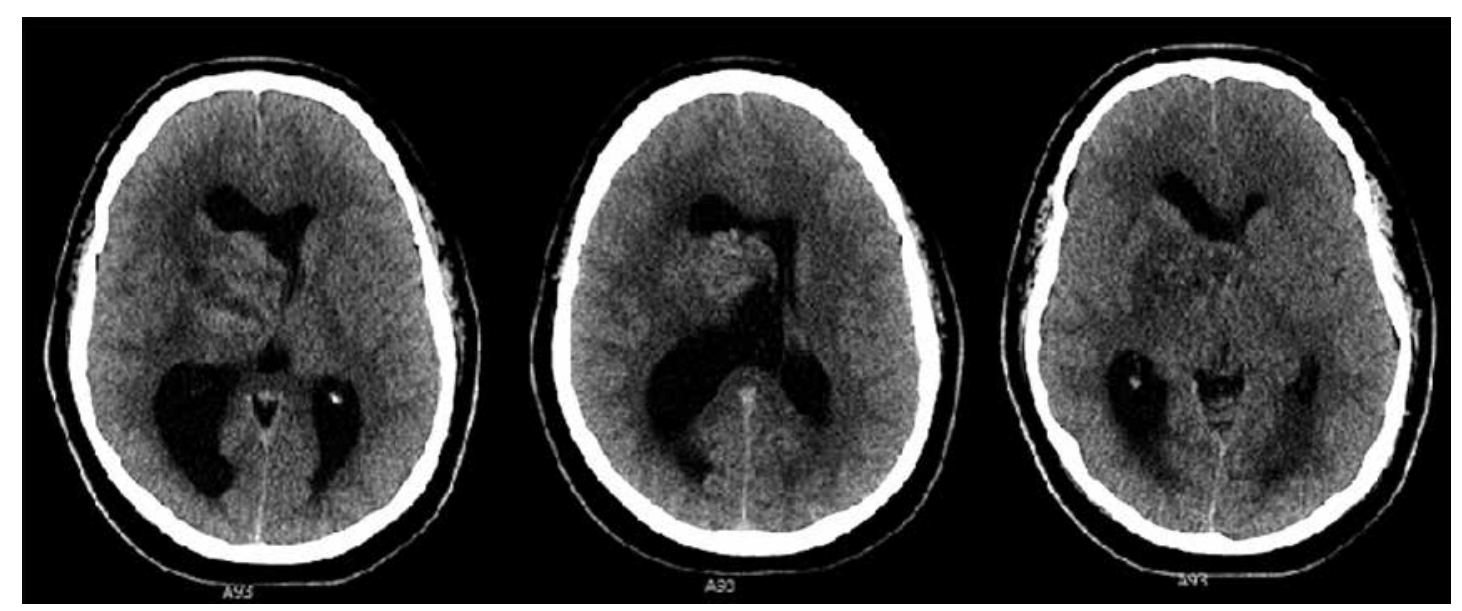

Fig. 1. Axial CT head, unenhanced. Heterogeneous mass in right thalamus and basal ganglia extending into the lateral ventricle. 

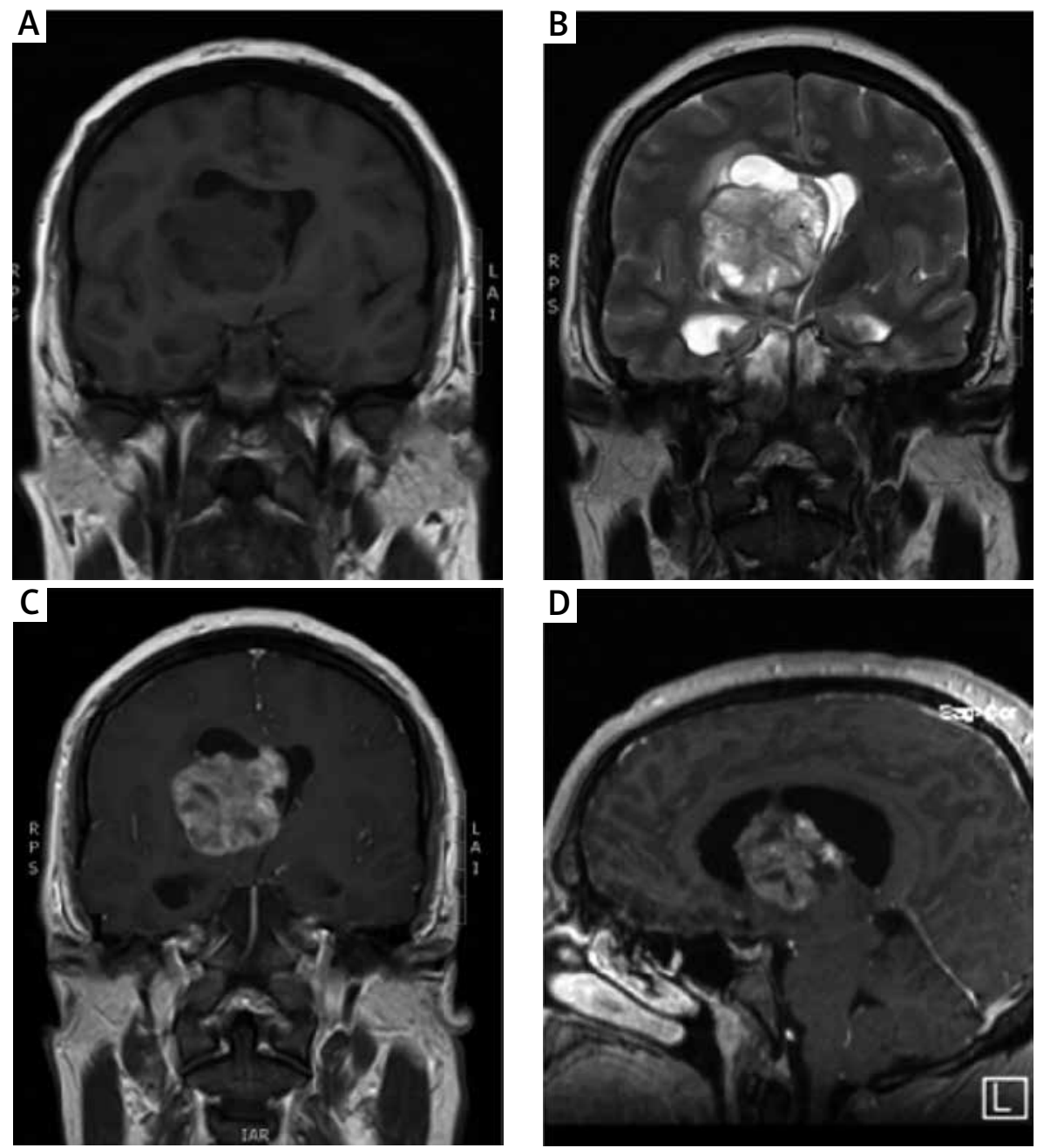

Fig. 2. MRI brain, unenhanced and enhanced. A) Coronal T1, unenhanced. Hypointense mass in right thalamus and basal ganglia, extending into the lateral ventricle. Necrotic/cystic areas within mass. B) Coronal T2, unenhanced. Heterogeneous mass within the lateral ventricle. C) T1 coronal, enhanced with Gadolinium and D) T1 sagittal, enhanced with Gadolinium. Heterogeneously enhancing lesion.

positivity for synaptophysin and EMA and patchy immunopositivity for GFAP (Fig. 4). ATRX immunohistochemistry showed retained nuclear expression by the tumour cells (Fig. 4). INI1 immunohistochemistry demonstrated diffuse loss of nuclear expression by the tumour cells Molecular panel, which included IDH and p53 did not reveal any mutations in IDH-1, IDH-2 or p53. $1 p / 19 q$ testing was not performed since there was no IDH mutation detected and oligodendroglioma was no longer a diagnostic consideration.

\section{Discussion}

AT/RT is defined by the presence of alterations of either INI1 protein (SMARCB1 gene), or rarely, BRG1 protein (SMARCA4 gene) [14]. Our literature review found 49 cases of AT/RT reported in adults with diagnosis confirmed with either INI1 or BRG1 alterations. Average age at diagnosis was 37 years [2]. Overall prognosis was poor, with an average overall survival of 20 months $[2,6,16]$. The cerebral hemispheres and the sellar region make up $78 \%$ of cases $[2,18]$. In contrast, the posterior fossa is the most common location for pediatric patients $[6,20]$. In both pediatric and adult patients, intraventricular location for AT/ RT is extremely rare [3]. To our knowledge, there are five previously reported cases in pediatric patients $[3,4,12,13,19]$. Our case report is the first reported case in an adult patient. The location provides chal- 

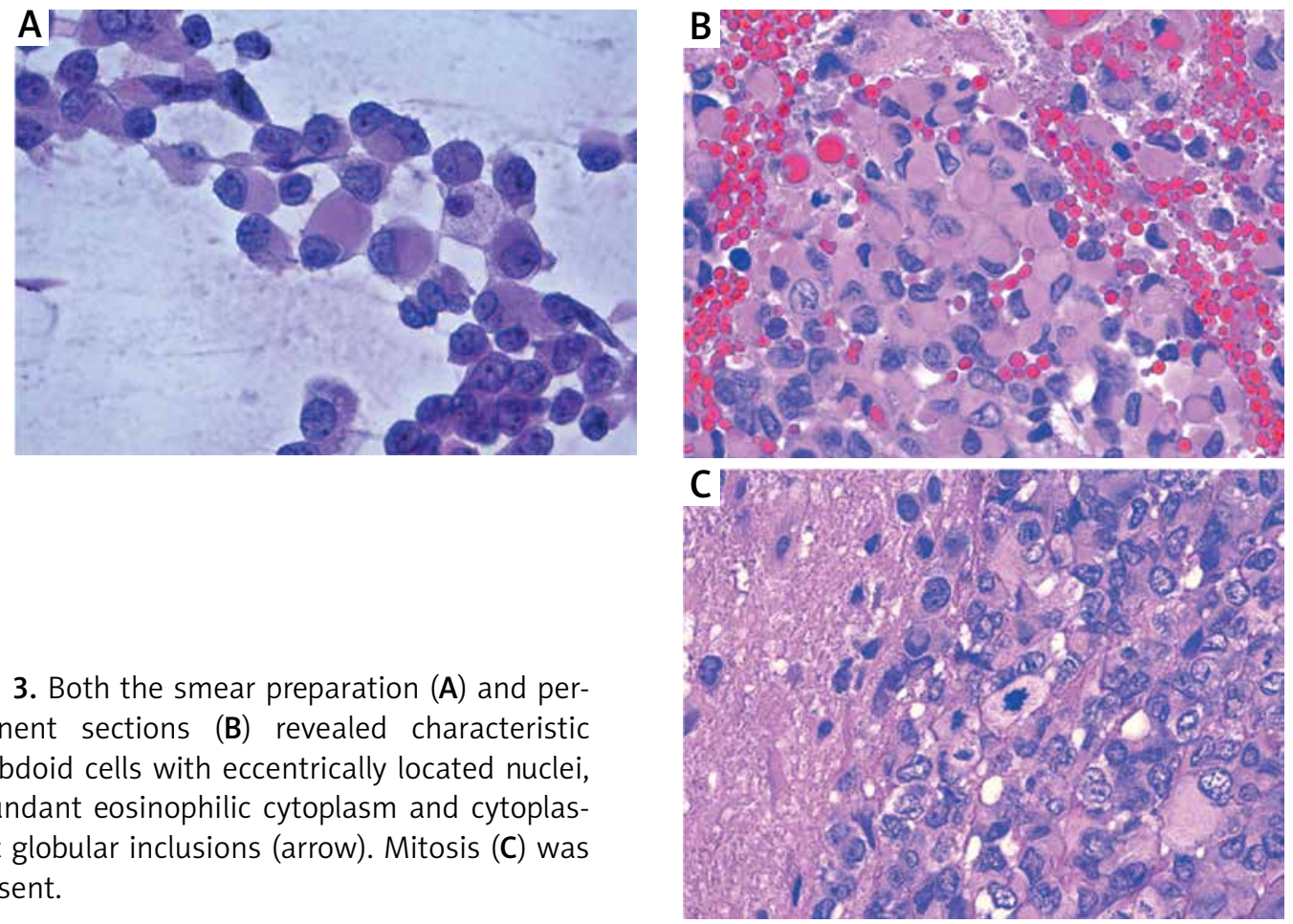

Fig. 3. Both the smear preparation (A) and permanent sections (B) revealed characteristic rhabdoid cells with eccentrically located nuclei, abundant eosinophilic cytoplasm and cytoplasmic globular inclusions (arrow). Mitosis (C) was present.

lenges to resection due to difficulty in visualizing the borders of the tumour, attachment to choroid plexus, large choroidal feeding arteries, and possible dissemination through the cerebrospinal fluid $[3,21]$. In our case, a gross total resection was not attained partly due to the location. The lateral edge of the tumour, within the left lateral ventricle, was difficult to visualize. Additionally, the tumour was extremely vascular, which made it difficult to visualize once there was blood within the cerebrospinal fluid.

As was the case in our patient, the most striking microscopic feature of AT/RT is the presence of rhabdoid cells with eccentrically located nuclei and abundant eosinophilic cytoplasm consisting of globular inclusions. Diagnosis of AT/RT in our patient was confirmed by loss of nuclear INI-1 expression. Recently three distinct molecular subgroups of AT/RT have been defined [8]. The TYR subgroup is characterized by broad SMARCB1 deletions and overexpression of melanosomal genes, the sonic-hedgehog $(\mathrm{SHH})$ subgroup is defined by focal SMARCB1 aberrations and overexpression of the SHH pathway, and MYC subgroup is defined by focal SMARCB1 deletions and overexpression of MYC and HOX cluster [8]. Nowak et al. performed a study on MRI findings in these molecular subgroups [17]. The authors observed more

pronounced peritumoral edema in the ATRT-MYC subgroups compared to the other two subgroups, while peritumoral cysts were more common in the ATRTSHH (71\%) and ATRT-TYR (94\%) subgroups. The study showed contrast enhancement to be absent in $29 \%$ of ATRT-SHH, $10 \%$ of ATRT-MYC, and none of the ATRTTYR cases. Rare examples of INI-1 loss in glioblastomas reported in the literature have shown focal INI-1 loss [10]. This differs from our case which exhibited diffuse loss of INI-1 expression by immunohistochemistry. Additionally, our tumour only showed patchy GFAP immunopositivity with the absence of microvascular proliferation and palisading necrosis, further arguing against glioblastoma with loss of INI-1.

Radiological findings from our case report were similar to those previously reported in adult AT/RT $[5,9]$. In our case, the tumour was hyperattenuated with cystic/necrotic components seen on CT. These features are seen in $91 \%$ of the cases of adult AT/ RT [9]. In a study by Kanoto et al., they reported $80 \%$ were hypointense on T1-weighted imaging, $72 \%$ were mixed-intensity on T2-weighted imaging, $100 \%$ had restricted diffusion, $70 \%$ had heterogeneous enhancement, $56 \%$ had cyst/necrosis, $42 \%$ had hemorrhage and 33\% had calcifications [9]. The areas of hemorrhage suggest a vascular tumour. 

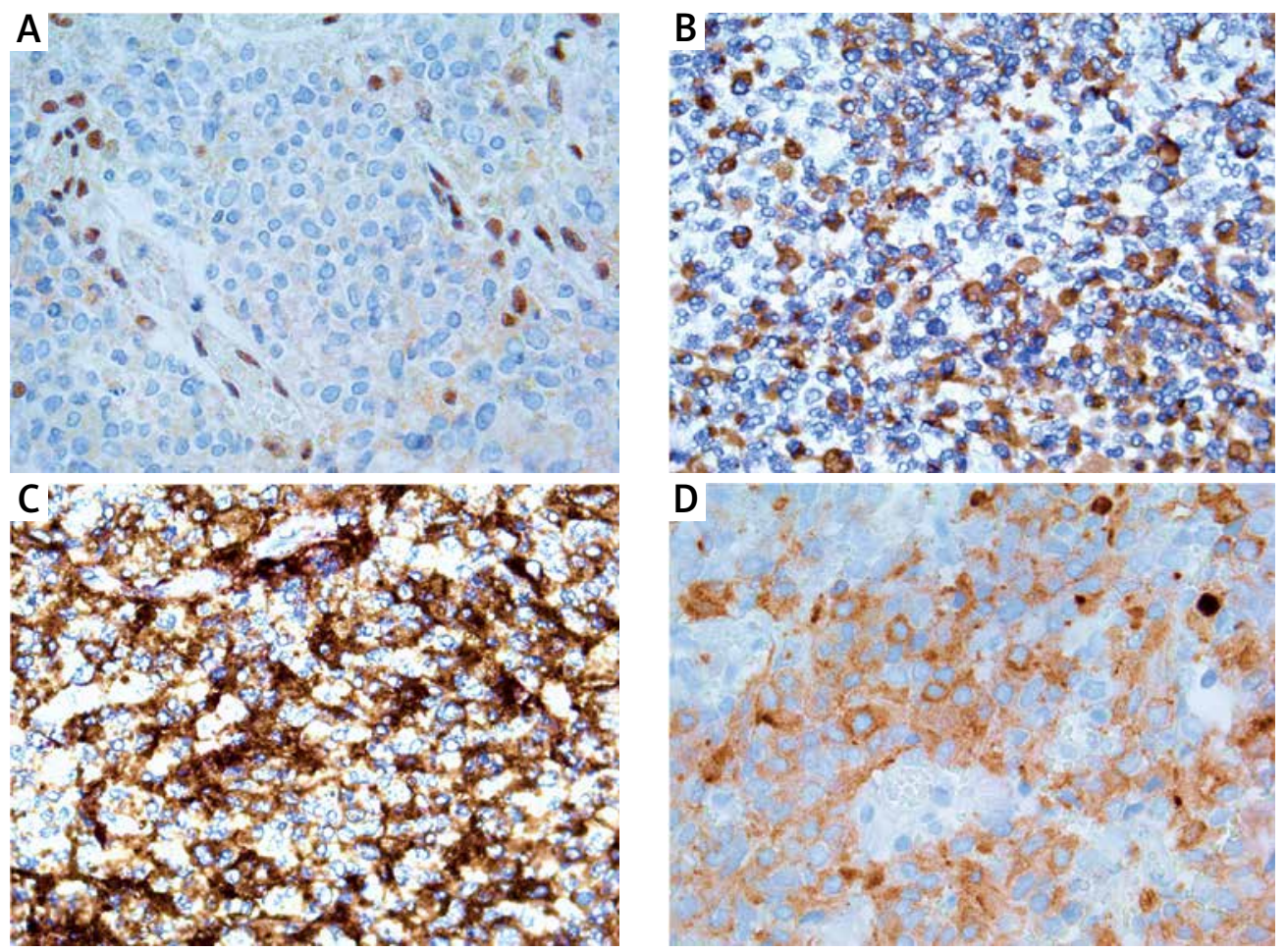

Fig. 4. INI-1 immunohistochemistry (A) demonstrated loss of nuclear expression. The tumour cells also showed patchy immunoreactivity for GFAP (B), EMA (C) and synaptophysin (D).

In one case study, cerebral angiography showed tumour stain and early venous filling suggestive of a hypervascular tumour [9]. These radiological findings of a vascular tumour were consistent with our intraoperative findings.

Multiple factors can affect the ability to completely resect a tumour including proximity to eloquent brain, involvement of surrounding neurovascular structures and vascularity. In our case, the vascular nature of the tumour limited the extent of resection achieved. In our literature review on AT/RT in adults, gross total resection was only achieved in $24 \%$ of cases [2]. Although the specific reasons for inability to achieve gross total resection was not specified, it is possible that vascularity was a limiting factor. One consideration for vascular intraaxial tumours could be preoperative embolization to increase the chances of achieving a gross total resection [1]. This has been done for other vascular intraaxial tumours, such as hemangioblastomas, metastatic lesions, and ependymomas [1]. In a case series on choroid plexus carcinoma, another hypervascular tumour, 4 out of 9 surgeries were abandoned due to excessive hemorrhage and gross total resection was only achieved in $33 \%[15,21]$. A case report on a choroid plexus carcino- ma describes the use of preoperative embolization to minimize blood loss and achieve good hemostasis after resection [21]. In a pediatric case report of an AT/RT in the lateral ventricle, a large feeding artery was clipped prior to resection to reduce blood loss [3]. There may be a role for angiography and preoperative embolization for AT/RT given the vascular nature of these tumours, in particular for tumours in difficult locations.

\section{Conclusions}

Herein we report a rare case of AT/RT in an adult patient that involved the lateral ventricle and deep grey matter. Radiological and intraoperative findings suggest AT/RT is a hypervascular tumour which may affect the ability to perform a complete resection and possibly increase the risk of postoperative bleeding, which was seen in our case. There may be thus a role for angiography and preoperative embolization in patients with AT/RT to improve the rate of gross total resection and hemostasis.

\section{Disclosure}

The authors report no conflict of interest. 


\section{References}

1. Bharadwaj S, Moga R, Manninen P, Venkatraghavan L. Anaesthetic considerations for patients undergoing pre-surgical embolization of intracranial and spinal tumours: An overview. J Neuroanaesthesiol Crit Care 2009; 2: 104-109.

2. Chan V, Marro A, Findlay JM, Schmitt LM, Das S. A Systematic review of atypical teratoid rhabdoid tumor in adults. Front Oncol 2018; 8: 567.

3. Darmoul M, Nsir AB, Hattab MN, Chabchoub I. Atypical teratoid rhabdoid tumor of the lateral ventricle. J Pediatr Neurosci 2015; 10: 382-385.

4. Donovan DJ, Smith AB, Petermann GW. Atypical teratoid/rhabdoid tumor of the velum interpositum presenting as a spontaneous intraventricular hemorrhage in an infant: case report with long-term survival. Pediatr Neurosurg 2006; 42: 187-192.

5. Han L, Qiu Y, Xie C, Zhang J, Lv X, Xiong W, Wang W, Zhang X, Wu P. Atypical teratoid/rhabdoid tumors in adult patients: CT and MR imaging features. Am J Neuroradiol 2011; 32: 103-108.

6. Hilden JM, Meerbaum S, Burger P, Finlay J, Janss A, Scheithauer BW, Walter AW, Rorke LB, Biegel JA. Central nervous system atypical teratoid/rhabdoid tumor: results of therapy in children enrolled in a registry. I Clin Oncol 2004; 22: 2877-2884.

7. Horiguchi H, Nakata S, Nobusawa S, Uyama S, Miyamoto T, Ueta H, Fujimoto N, Yokoo H. Adult onset atypical teratoid/rhabdoid tumor featuring long spindle cells with nuclear palisading and perivascular pseudorosettes. Neuropathology 2017; 37: 52-57.

8. Johann PD, Erkek S, Zapatka M, Kerl K, Buchhalter I, Hovestadt V, Jones DT, Sturm D, Hermann C, Wang MA, Korshunov A, Rhyzova M, Gröbner S, Brabetz S, Chavez L, Bens S, Gröschel S, Kratochwil F, Wittmann A, Sieber L, Geörg C, Wolf S, Beck K, Oyen F, Capper D van Sluis P, Volckmann R, Koster J, Versteeg R, von Deimling A, Milde T, Witt O, Kulozik AE, Ebinger M, Shalaby T, Grotzer M. Atypical teratoid/rhabdoid tumors are comprised of three epigenetic subgroups with distinct enhancer landscapes. Cancer Cell 2016; 29: 379-393.

9. Kanoto M, Toyoguchi Y, Hosoya T, Kuchiki M, Sugai Y. Radiological image features of the atypical teratoid/rhabdoid tumor in adults: a systematic review. Clin Neuroradiol 2015; 25: 55-60.

10. Kleinschmidt-DeMasters BK, Alassiri AH, Birks DK, Newell KL, Moore W, Lillehei KO. Epithelioid versus rhabdoid glioblastomas are distinguished by monosomy 22 and immunohistochemical expression of INI-1 but not claudin 6. Am J Surg Pathol 2010; 34: 341-354.

11. Lee J, Kim DS, Han JW, Suh CO. Atypical teratoid/rhabdoid tumors in children treated with multimodal therapies: The necessity of upfront radiotherapy after surgery. Pediatr Blood Cancer 2017; 64: e26663.

12. Lee IH, Yoo SY, Kim JH, Eo H, Kim OH, Kim IO, Cheon JE, Jung AY, Yoon BJ. Atypical teratoid/rhabdoid tumors of the central nervous system: imaging and clinical findings in 16 children. Clin Radiol 2009; 64: 256-264.

13. Li F, Gui Q, Piao Y. Primary supratentorial atypical teratoid/rhabdoid tumor in children: a report of two cases. I Child Neurol 2013; 28: 399-403.

14. Louis DN, Perry A, Reifenberger G, Von Deimling A, Figarella-Branger D, Cavenee WK, Ohgaki H, Wiestler OD, Kleihues P,
Ellison DW. The 2016 World Health Organization classification of tumors of the central nervous system: a summary. Acta Neuropathol 2016; 131: 803-820.

15. McEvoy AW, Harding BN, Phipps KP, Ellison DW, Elsmore AJ, Thompson D, Harkness W, Hayward RD. Management of choroid plexus tumours in children: 20 years experience at a single neurosurgical centre. Pediatr Neurosurg 2000; 32: 192-199.

16. Nakata S, Nobusawa S, Hirose T, Ito S, Inoshita N, Ichi S, Amatya VJ, Takeshima Y, Sugiyama K, Sonoda Y, Haga H, Hirato J, Nakazato Y, Yokoo H. Sellar atypical teratoid/rhabdoid tumor (AT/RT). A clinicopathologically and genetically distinct variant of AT/RT. Am J Surg Pathol 2017; 41: 932-940.

17. Nowak J, Nemes K, Hohm A, Vandergrift LA, Hasselblatt M, Johann PD, Kool M, Frühwald MC, Warmuth-Metz M. Magnetic resonance imaging surrogates of molecular subgroups in atypical teratoid/rhabdoid tumor. Neuro Oncol 2018; 20: 1672-1679.

18. Ostrom QT, Chen Y, M de Blank P, Ondracek A, Farah P, Gittleman H, Wolinsky Y, Kruchko C, Cohen ML, Brat DJ, Barnholtz-Sloan JS. The descriptive epidemiology of atypical teratoid/rhabdoid tumors in the United States, 2001-2010. Neuro Oncol 2014; 16: 1392-1399.

19. Parwani AV, Stelow EB, Pambuccian SE, Burger PC, Ali SZ. Atypical teratoid/rhabdoid tumor of the brain: cytopathologic characteristics and differential diagnosis. Cancer 2005; 105: 65-70.

20. Rorke LB, Packer RJ, Biegel JA. Central nervous system atypical teratoid/rhabdoid tumors of infancy and childhood: definition of an entity. J Neurosurg 1996; 85: 56-65.

21. Slater LA, Hoffman C, Drake J, Krings T. Pre-operative embolization of a choroid plexus carcinoma: Review of the vascular anatomy. Childs Nerv Syst 2016; 32: 541-545. 